Вестник Новосибирского государственного педагогического университета

2018, том 8, № 3

www.vestnik.nspu.ru

ISSN 2226-3365

(C) И. Ф. Харина, Е. В. Звягина, Е. В. Быков, О. А. Макунина

DOI: $10.15293 / 2226-3365.1803 .13$

УДК 612.8+159.952

\title{
ОСОБЕННОСТИ ПСИХОФИЗИОЛОГИЧЕСКИХ ПОКАЗАТЕЛЕЙ СТУДЕНТОВ
}

\section{С ПРИЗНАКАМИ ДЕФИЦИТА ВНИМАНИЯ В УСЛОВИЯХ СОЧЕТАННЫХ УМСТВЕННЫХ И ФИЗИЧЕСКИХ НАГРУЗОК*}

\author{
И. Ф. Харина, Е. В. Звягина, Е. В. Быков, О. А. Макунина (Челябинск, Россия)
}

Проблема и цель. Студенты с признаками дефицита внимания отличаются психофизиологическими особенностями, особенно необходимыми в условиях сочетанных умственных и физических нагрузок. В статье представлены результаты исследования по изучению особенностей психофизиологических показателей у студентов с признаками дефицита внимания университета физической культуры. Целью исследования является изучение психофизиологических особенностей и адаптаџии студентов с признаками дефищита внимания, обучающихся в университете физической культуры.

Методология. Исследование проводилось с помощью теста Тулуз-Пьерона, который является одним из вариантов корректурной пробы, общий принцип которого сводится к дифференцированию стимулов, близких по форме и содержанию в течение точно определенного времени. Первично оцениваются свойства внимания (концентрации, устойчивости, переключаемости), вторично - точность и надежность переработки информации. Всего обследовано 154 студента I-III курсов. Психофизиологические показатели оценивали методиками ПЗМР и теппинг-тестом. Оиенку адаптаџии определяли по формуле Р. М. Баевского.

* Статья подготовлена в рамках выполнения проекта государственного задания «Мониторинг состояния здоровья студентов университета физической культуры г. Челябинска».

Харина Ирина Федоровна - кандидат педагогических наук, доцент, кафедра анатомии, Уральский государственный университет физической культуры, научный сотрудник НИИ ОС.

E-mail: kharina.i.f@list.ru

Звягина Екатерина Владимировна - кандидат педагогических наук, доцент, кафедра анатомии, Уральский государственный университет физической культуры, научный сотрудник НИИ ОС.

E-mail: zv-aev@mail.ru

Быков Евгений Витальевич - доктор медицинских наук, профессор, заведующий кафедрой спортивной медицины, проректор по науке, Уральский государственный университет физической культуры.

E-mail: bev58@yandex.ru

Макунина Ольга Александровна - кандидат биологических наук, доцент, кафедра физиологии, Уральский государственный университет физической культуры, старший научный сотрудник НИИ ОС.

E-mail: oamakunina@mail.ru 
Вестник Новосибирского государственного педагогического университета

2018, том 8, № 3

www.vestnik.nspu.ru

ISSN 2226-3365

Результаты. В ходе исследования установлено, что скорость работы студентов не соответствует возрастным нормам: низкая скорость определена у 35,06 \% студентов; средняя скорость характерна для 45,45 \% студентов; наличие признаков дефицитта внимания отмечается у студентов II и III курсов обучения и составляет 8,82 и 9,62\% соответственно. Высокая точность выявлена у 44,16\% (68 студентов).

Психофизиологические характеристики обследуемых студентов соответствуют возрастным нормам и не имеют различий между студентами в зависимости от свойств внимания. Однако значимый разброс коэффициентов вариации психофизиологических показателей может свидетельствовать о разных причинах дефицита внимания студентов. Студенты с признаками дефищита внимания испытывают напряжение механизмов адаптации.

Заключение. В статье предложены рекомендации для студентов и преподавателей, способствующие усвоению учебного материала и повышению качества обучения. Исследование будет продолжено в аспекте выявления причин дефицита внимания и изучения нейрофизиологических особенностей у студентов в условиях сочетанных умственных и физических нагрузок.

Ключевые слова: мониторинг здоровья; дефицит внимания; свойства внимания; студентыл-спортсмены; тест Тулуз-Пьерона; адаптация; психофизиологические особенности.

\section{Постановка проблемы}

Современные исследования в области изучения свойств внимания и выявления признаков синдрома дефицита внимания и гиперактивности (СДВГ) у студенческой молодежи и взрослых направлены на подтверждение этого диагноза в данной группе [1-6]. Исследованиями И. Т. Нуреева, О. В. Поповой и других ученых было установлено, что среди студентов педагогического вуза г. Кирова синдром дефицита внимания встречается у 15,7 \% (24,0 \% - юноши, 12,1 \% - девушки) и у $8,8 \%$ студентов медицинского вуза г. Кирова $(8,9 \%$ - это юноши и $8,7 \%$ - девушки) [7-9]. Е. В. Жинжило и другие при анонимном анкетировании студентов вузов г. Ростова-наДону диагностировали этот синдром у $11 \%$ обучающихся (6 \% - юноши и $12,5 \%$ - девушки) [10]. Исследования, проведенные нами ранее на контингенте студентов колледжа
УралГУФК (Челябинск), позволили установить, что у $11,76 \%$ студентов имеют дефицит внимания [11-13].

В зарубежных научных изданиях результаты исследований по изучению психофизиологических особенностей студентов с признаками дефицита внимания мы не обнаружили. Однако при анализе статей по этой проблеме мы выделили несколько направлений: 1) изучение генетических причин СДВГ [14]; 2) апробация фармакологических способов лечения СДВГ [15-17]; 3) нейропсихологическая оценка популяции детей и молодежи с СДВГ [5; 18; 19]; 4) клиническое применение биоуправления в лечении СДВГ [20; 21]; 5) разработка комплекса мероприятий образовательной деятельности учащихся с дефицитом внимания [22; 23].

За последнее десятилетие в 6 раз увеличилось количество научных исследований по применению различных вариантов нейробиоуправления в лечении диагноза СДВГ. В работе 
[24] представлен обзор литературы за последние 10 лет об использовании метода нейробиоуправления при лечении СДВГ. Л. С. Чутко с соавторами представляют в своих работах положительные результаты исследования диадного подхода (одновременного лечения матерей и детей) и терапевтического эффекта препаратов на разных категориях обследуемых подростков и взрослых [25].

Результаты исследований, представленные в зарубежных и российских публикациях, подтверждают актуальность обозначенной темы и свидетельствуют о недостаточном изучении психофизиологических особенностей и механизмов адаптации студентов с признаками дефицита внимания. Свойства внимания являются важной психофизиологической характеристикой личности. От уровня свойств внимания зависит физиологическая цена адаптации организма к предъявляемым нагрузкам. Особенно важна ценность этих свойств в условиях сочетанных умственных и физических нагрузок. В таких условиях студенты-спортсмены должны обладать высокими показателями концентрации, скорости, точности внимания. Мы полагаем, что при низких значениях внимания и признаков дефицита внимания организм студента-спортсмена испытывает напряжение физиологических систем адаптации. Из этого следует, что изучение особенностей внимания и выявление признаков дефицита внимания у студентов в условиях сочетанных умственных и физических нагрузок является актуальным и дополняет комплекс методов по оценке адаптации студентов-спортсменов к предъявляемым нагрузкам.

${ }^{1}$ Шибкова Д. 3., Смирнова Ю. В., Макунина О. А. Управление качеством образования на основе мониторинга здоровья учащихся. - Челябинск, 2007. 364 c. URL: https://elibrary.ru/item.asp?id=21531815
Изучение свойств внимания и выявление студентов с признаками дефицита внимания было включено в программу мониторинга здоровья студентов Уральского государственного университета физической культуры [26; 27]. Мониторинг состояния здоровья студентов позволяет дать оценку эффективности здоровьесберегающей деятельности в системе образования ${ }^{1}$ [28]. Целью исследования является изучение психофизиологических особенностей и адаптации студентов с признаками дефицита внимания, обучающихся в университете физической культуры.

\section{Материалы и методы исследования}

Исследование было проведено в начале 2017/2018 учебного года в рамках психофизиологического блока мониторинга здоровья студентов УралГУФК с соблюдением всех этических норм и требований, предъявляемых к медико-биологическим исследованиям. В исследовании приняли участие 154 студента, из них 34 студента I курса, 68 студентов II курса и 52 студента III курса, средний возраст составил 19,0 \pm 0,5 лет. Все студенты были уведомлены о цели исследования и дали добровольное письменное согласие на участие.

Студентам было предложено выполнить тест Тулуз-Пьерона. Он направлен на изучение свойств внимания, основными показателями являются коэффициент точности или показатель концентрации внимания $(K$ - отношение правильно обработанных знаков к общему количеству знаков) и интегральный показатель скорости ( $V$ - количество обработанных знаков в минуту). Согласно исследованиям Л. А. Ясюковой ${ }^{2}$ на взрослой выборке результаты тестирования стабильны во времени,

\footnotetext{
2 Ясюкова Л. А. Оптимизация обучения и развития детей с ММД. Диагностика и компенсация минимальных мозговых дисфункций: методическое руководство. - СПб.: ИМАТОН, 1997. - 80 с.
} 
кроме этого один из показателей теста - это скорость (знаков/мин), которая связана с возрастным созреванием нервной системы. Данные характеристики позволили использовать эту методику в возрастной группе студентов I-III курсов.

Тест применяется как экспресс-метод косвенной диагностики малой мозговой дисфункции (ММД) (согласно современным трактовкам СДВГ). Принимая во внимание, что окончательный диагноз ставится неврологом, мы ставим перед собой задачу при выявлении у студентов признаков дефицита внимания разработать рекомендации по здоровому образу жизни и тренировочно-соревновательному режиму с учетом особенностей свойств внимания.

Перед началом психофизиологических обследований проводили анамнез студентов о текущем самочувствии, о наличии тренировок. Студенты приглашались в лабораторию на обследование через день после тренировок во время естественной учебной деятельности в университете. Полагаем, что такой подход позволяет объективно оценить реакцию систем жизнеобеспечения в условиях сочетанных нагрузок студентов-спортсменов. Оценку психофизиологических показателей студентов проводили общеизвестными методиками ПЗМР и теппинг-тестом АПК «Психотест» $(\mathrm{OOО} \mathrm{«Нейрософт»,} \mathrm{г.} \mathrm{Иваново})^{3}$. Оценку адаптационного потенциала (АП) проводили по формуле Р. М. Баевского 4 :

$\mathrm{AП}=0,011 \times$ ЧП + 0,014 $\times$ АДс $+0,008 \times$ АДд $+0,014 \times \mathrm{B}+0,009 \times \mathrm{MT}-0,009 \times \mathrm{P}-0,27$,

где АП - адаптационный потенциал;
В - возраст, лет;

МТ - масса тела, кг;

$\mathrm{P}$ - рост, см;

АДс - артериальное давление систолическое, мм рт. ст.;

АДд - артериальное давление диастолическое, мм рт. ст.;

ЧП - частота пульса за 1 минуту.

Статистический анализ полученных данных осуществлялся посредством программы Microsoft Office Excel, проводили расчет средней арифметической величины (M), стандартного отклонения $( \pm \sigma)$ и ошибки средней арифметической величины $( \pm \mathrm{m})$, оценка достоверности различий производилась с помощью $t$-критерия Стьюдента. Для анализа психофизиологических показателей проводили оценку степени рассеивания показателей в популяции по коэффициенту вариации. Этот показатель измеряется в процентах. В статистике принято, что, если коэффициент вариации меньше $10 \%$, то степень рассеивания данных считается незначительной, от 10 до $20 \%$ средней, больше $20 \%$ и меньше или равно 33 \% - значительной, значение коэффициента вариации не превышает $33 \%$, то совокупность считается однородной, если больше $33 \%$, то неоднородной.

\section{Результаты исследования}

В таблице 1 представлены среднестатистические показатели скорости, количества ошибок и точности выполнения теста ТулузПьерона (по Л. А. Ясюковой) у студентов I-III курсов УралГУФК.

\footnotetext{
${ }^{3}$ Мантрова И. Н. Методическое руководство по психофизиологической и психологической диагностике. - Иваново: Нейрософт, 2007. - 216 с.
} 
Вестник Новосибирского государственного педагогического университета

2018, том 8, № 3

www.vestnik.nspu.ru

ISSN 2226-3365

Таблица 1

Среднестатистические показатели скорости, количества ошибок и точности выполнения теста Тулуз-Пьерона (по Л. А. Ясюковой) у студентов I-III курсов УралГУФК

Table 1

Overall average of speed, mistakes number and accuracy of Toulouse -Perron test performing (by L. A. Yasyukova) of I-III year students of the Ural State University of Physical Culture («UralSUPC»)

\begin{tabular}{|c|c|c|c|c|c|c|c|c|c|c|}
\hline \multirow[t]{2}{*}{ Курс/пол } & \multirow[t]{2}{*}{$n$} & \multicolumn{3}{|c|}{$\begin{array}{l}\text { Скорость (V) } \\
\text { (знаков/мин) }\end{array}$} & \multicolumn{3}{|c|}{$\begin{array}{c}\text { Среднее количество } \\
\text { ошибок (а) }\end{array}$} & \multicolumn{3}{|c|}{$\begin{array}{c}\text { Точность (К) } \\
\text { (усл. ед.) }\end{array}$} \\
\hline & & $\mathrm{M}$ & $\pm \sigma$ & $\pm \mathrm{m}$ & $\mathrm{M}$ & $\pm \sigma$ & $\pm \mathrm{m}$ & $\mathrm{M}$ & $\pm \sigma$ & $\pm \mathrm{m}$ \\
\hline I курс & 34 & 54,12 & $\pm 11,24$ & $\pm 1,96$ & 1,77 & $\pm 1,27$ & $\pm 0,22$ & 0,97 & $\pm 0,02$ & $\pm 0,00$ \\
\hline Девушки & 26 & $51,48 * *$ & $\pm 8,94$ & $\pm 1,79$ & 1,55 & $\pm 0,97$ & $\pm 0,19$ & 0,97 & $\pm 0,02$ & $\pm 0,00$ \\
\hline Юноши & 8 & $62,70 * *$ & $\pm 14,14$ & $\pm 5,34$ & 2,50 & $\pm 1,85$ & $\pm 0,70$ & 0,96 & $\pm 0,03$ & $\pm 0,01$ \\
\hline II курс & 68 & 51,31 & $\pm 10,86$ & $\pm 1,33$ & 2,08 & $\pm 2,64$ & 0,32 & 0,95 & $\pm 0,06$ & $\pm 0,01$ \\
\hline Девушки & 31 & 49,61 & $\pm 10,40$ & $\pm 1,90$ & 1,44 & $\pm 1,18$ & $\pm 0,22$ & 0,97 & $\pm 0,03$ & $\pm 0,01$ \\
\hline Юноши & 37 & 52,75 & $\pm 11,17$ & $\pm 1,86$ & 2,61 & $\pm 3,34$ & $\pm 0,56$ & 0,94 & $\pm 0,07$ & $\pm 0,01$ \\
\hline III курс & 52 & 52,73 & $\pm 11,22$ & $\pm 1,57$ & 2,06 & $\pm 2,11$ & $\pm 0,30$ & 0,96 & $\pm 0,04$ & $\pm 0,01$ \\
\hline Девушки & 22 & 52,79 & $\pm 8,69$ & $\pm 1,90$ & 1,80 & $\pm 1,96$ & $\pm 0,43$ & 0,96 & $\pm 0,03$ & $\pm 0,01$ \\
\hline Юноши & 30 & 52,68 & $\pm 12,91$ & $\pm 2,40$ & 2,24 & $\pm 2,23$ & $\pm 0,41$ & 0,95 & $\pm 0,04$ & $\pm 0,01$ \\
\hline $\begin{array}{l}\text { ВСЕ } \\
\text { участники } \\
\text { исследования }\end{array}$ & 154 & 52,41 & $\pm 11,05$ & $\pm 0,89$ & 2,0 & $\pm 2,21$ & $\pm 0,18$ & 0,96 & $\pm 0,04$ & $\pm 0,00$ \\
\hline Девушки & 79 & $51,11^{*}$ & $\pm 9,45$ & $\pm 1,07$ & 1,58 & $\pm 1,38$ & $\pm 0,16$ & 0,97 & $\pm 0,03$ & $\pm 0,00$ \\
\hline Юноши & 75 & $53,78^{*}$ & $\pm 12,43$ & $\pm 1,45$ & 2,45 & $\pm 2,78$ & $\pm 0,32$ & 0,95 & $\pm 0,06$ & $\pm 0,01$ \\
\hline
\end{tabular}

Мы выявили особенности динамики свойств внимания у студентов на разных курсах обучения. Так, студенты I курса характеризуются низкими значениями скорости, но высокими значениями качества (количество ошибок) работы. У студентов II курса скорость работы увеличивается на 5,2 \% при ухудшении качества работы на 17,5\%. К III курсу отмечается некоторое уменьшение скорости работы на 2,8 \% при неизменном качестве (количество ошибок изменилось на $0,9 \%)$.

У 34 студентов I курса показатели скорости $(\mathrm{M} \pm \mathrm{m})$ составили $54,12 \pm 1,96$ знаков/мин, эти данные соответствует средним значениям возрастных нормативов выполнения теста Ту-
луз-Пьерона. Полученные результаты, предположительно, объясняются наличием в одной из учебных групп студента (реализующегося в киберспорте), выполнившего тест с максимальной скоростью $(89,4$ знаков/мин), хотя точность выполнения теста была в пределах средних показателей (0,95 усл. ед.).

Показатели скорости в исследуемой группе студентов II курса $(n=68)$ составили $51,31 \pm 1,33$ знаков/мин, при этом у юношей $52,75 \pm 1,86$ знаков/мин, что соответствует средним значениям, тогда как у девушек несколько ниже 49,61 $\pm 1,90$ знаков/мин. Значения точности выполнения теста $(\mathrm{M} \pm \mathrm{m})$ у студентов II курса УралГУФК составили 0,95 \pm 0,01 при этом у студенток $0,97 \pm 0,01$ усл. ед., что соот- 
Вестник Новосибирского государственного педагогического университета

ветствует хорошей точности, тогда как у юношей полученные значения $(0,94 \pm 0,01$ усл. ед.) попадают в пределы средних возрастных нормативов выполнения этого теста.

Студентов III курса было обследовано 52 человека, из них 22 девушки и 30 юношей. Выявленные значения по скорости выполнения теста $52,73 \pm 1,57$ знаков/мин находятся в пределах средних показателей, у девушек и юношей значения оказались близкими $(52,79 \pm 1,90$ и $52,68 \pm 2,40$ знаков/мин). Студенты выполнили тест с точностью $0,95 \pm 0,01$ усл. ед. (средние показатели по возрастным нормативам), студентки $-0,96 \pm 0,01$ усл. ед. (хорошая точность). Значения точности выполнения теста $(\mathrm{M} \pm \mathrm{m})$ у студентов III курса УралГУФК составили $0,96 \pm 0,01$ усл. ед.
Итак, студенты Уральского государственного университета физической культуры показывают преимущественно среднюю скорость $52,41 \pm 0,89$ знаков/мин и хорошую точность $0,96 \pm 0,00$ усл. ед. выполнения данной методики экспресс-диагностики свойств внимания.

Для выявления признаков дефицита внимания особое значение имеет показатель коэффициента точности (K, усл. ед.) выполнения теста Тулуз-Пьерона, который в первую очередь характеризует концентрацию внимания.

В таблице 2 представлено распределение обследуемых студентов по уровням показателя коэффициента точности.

Таблицьа 2

Распределение студентов I-III курсов УралГУФК по уровням показателя точности (К, усл. ед.) выполнения теста Тулуз-Пьерона (по Л. А. Ясюковой)

Table 2

I-III year students of the Ural State University of Physical Culture («UralSUPC») distribution according to accuracy characteristics levels (A, nominal units) of Toulouse-Perron test performing (by L. A. Yasyukova)

\begin{tabular}{|l|l|c|c|c|c|c|}
\hline Курс/пол & $\boldsymbol{n}$ & $\begin{array}{c}\text { Патология } \\
\mathbf{( 0 , 9} \mathbf{~ и ~ < ) , ~ \% ~}\end{array}$ & $\begin{array}{c}\text { Слабая } \\
\mathbf{( 0 , 9 1 - 0 , 9 2 ) , ~ \% ~}\end{array}$ & $\begin{array}{c}\text { Средняя } \\
\mathbf{( 0 , 9 3 - 0 , 9 5 ) , ~ \% ~}\end{array}$ & $\begin{array}{c}\text { Хорошая } \\
\mathbf{( 0 , 9 6 - 0 , 9 7 ) , ~ \% ~}\end{array}$ & $\begin{array}{c}\text { Высокая } \\
\mathbf{( 0 , 9 8 - 1 ) , ~ \% ~}\end{array}$ \\
\hline I курс & 34 & 0 & 5,88 & 20,59 & 29,41 & 44,12 \\
\hline Девушки & 26 & 0 & 3,85 & 15,38 & 38,46 & 42,31 \\
\hline Юноши & 8 & 0 & 12,5 & 37,5 & 0 & 50 \\
\hline II курс & 68 & 8,82 & 7,35 & 16,18 & 19,12 & 48,53 \\
\hline Девушки & 31 & 6,46 & 3,22 & 9,68 & 22,58 & 58,06 \\
\hline Юноши & 37 & 10,81 & 10,81 & 21,62 & 16,22 & 40,54 \\
\hline III курс & 52 & 9,62 & 5,77 & 13,46 & 32,69 & 38,46 \\
\hline Девушки & 22 & 9,10 & 0 & 13,64 & 40,90 & 36,36 \\
\hline Юноши & 30 & 10,00 & 10,00 & 13,33 & 26,67 & 40,00 \\
\hline Все & 154 & 7,14 & 6,49 & 16,23 & 25,98 & 44,16 \\
\hline Девушки & 79 & 5,06 & 2,53 & 12,66 & 32,91 & 46,84 \\
\hline Юноши & 75 & 9,33 & 10,67 & 20,00 & 18,67 & 41,33 \\
\hline
\end{tabular}

Анализ показателя точности позволил установить, что в исследуемой группе 7,14 \% студентов имеют патологические значения и
6,49 \% студентов имеют слабый уровень значений. На первом курсе студентов с патологическими показателями не выявлено. На втором курсе 8,82 \% (6,46 \% девушек и 10,81\% 
Вестник Новосибирского государственного педагогического университета

юношей), на третьем курсе 9,62 \% студентов $(9,10 \%$ - девушки и 10,00 \% - юноши) имеют патологические значения.
Распределение обследуемых по уровням интегрального показателя скорости выполнения теста $(V$, знаков/мин) представлено в таблице 3.

Таблица 3

Распределение студентов I-III курсов УралГУФК по уровням интегрального показателя скорости (V, знаков/мин) выполнения теста Тулуз-Пьерона (по Л. А. Ясюковой)

Table 3

I-III year students of the Ural State University of Physical Culture («UralSUPC») distribution according to speed integral index levels $(V$, signs/min) of Toulouse-Perron test performing

(by L. A. Yasyukova)

\begin{tabular}{|l|c|c|c|c|c|}
\hline \multicolumn{1}{|c|}{ Курс/пол } & $\mathbf{n}$ & $\begin{array}{c}\text { Слабая } \\
\mathbf{( 4 9} \text { и <), \% }\end{array}$ & $\begin{array}{c}\text { Средняя } \\
\mathbf{( 5 0 - 6 2 ) ,} \%\end{array}$ & $\begin{array}{c}\text { Хорошая } \\
\mathbf{( 6 3 - 7 7 ) , ~ \% ~}\end{array}$ & $\begin{array}{c}\text { Высокая } \\
\mathbf{( 7 8} \text { и >), \% }\end{array}$ \\
\hline I курс & 34 & 29,41 & 47,05 & 20,59 & 2,95 \\
\hline Девушки & 26 & 34,61 & 50,00 & 15,39 & 0 \\
\hline Юноши & 8 & 12,5 & 37,5 & 37,5 & 12,5 \\
\hline II курс & 68 & 39,70 & 42,65 & 16,18 & 1,47 \\
\hline Девушки & 31 & 48,38 & 41,94 & 6,46 & 3,22 \\
\hline Юноши & 37 & 32,44 & 43,24 & 24,32 & 0 \\
\hline III курс & 52 & 32,69 & 48,08 & 17,31 & 1,92 \\
\hline Девушки & 22 & 27,27 & 59,09 & 13,64 & 0 \\
\hline Юноши & 30 & 36,67 & 40,0 & 20,0 & 3,33 \\
\hline Все & 154 & 35,06 & 45,45 & 17,54 & 1,95 \\
\hline Девушки & 79 & 37,97 & 49,37 & 11,39 & 1,27 \\
\hline Юноши & 75 & 32,00 & 41,33 & 24,00 & 2,67 \\
\hline
\end{tabular}

По результатам исследований в рамках мониторинга здоровья студентов УралГУФК мы выявили 35,06 \% обучающихся, выполняющих тест Тулуз-Пьерона со слабой скоростью, при этом среди девушек данная группа составила 37,97 \%, а среди юношей 32,00 \%. Детальный анализ показал, что количество студентов составило на первом курсе $29,41 \%$ (девушки - 34,61 \%, юноши - 12,5 \%), на втором курсе - 39,70 \% (девушки - 48,38 \%, юноши - 32,44 \%), на третьем курсе - 32,69 \% (девушки - 27,72 \%, юноши - 36,67 \%). Также мы выявили крайние значения по скорости выполнения теста: крайне низкая скорость определена у 7,79 \% (12 человек), тогда как показатели точности выполнения теста у данных респондентов от слабых до высоких, что подтверждают литературные данные и собственные изыскания о наличии признаков дефицита внимания у студенческой молодежи. Однако эта группа студентов должна пройти более углубленные исследования с использованием медико-психолого-педагогических методик, а также получить консультацию невропатолога.

В заключение этого этапа нашего исследования отметим, что скорость работы студентов не соответствует возрастным нормам: низкая скорость определена у 54 студентов $35,06 \%$; средняя скорость характерна для 70 студентов - 45,45 \%, хорошая скорость у 27 студентов - 17,54 \%. Девушки чаще показывают низкую и среднюю скорость 37,97 \% 
(30 студенток) и 49,37 \% (39 студенток соответственно); у юношей данные значения характерны для 32,00 \% (24 студентов) и 41,33 \% (31 студентов соответственно); показатели хорошей и высокой скорости у юношей выше, чем у девушек: $24,00 \%$ (18 студентов) и 2,67 \% (2 студента); 11,39\% (9 студенток) и 1,27 \% (1 студентка). Полученные результаты отражают психофизиологические особенности студентов-спортсменов.

Такая же неблагоприятная тенденция выражается и при анализе скорости выполнения теста по курсам: на I курсе низкая скорость определена для 29,41 \% студентов, на II курсе - у 39,70 \% респондентов, на III курсе

- 32,69 \%. Высокую скорость проявили по одному студенту I, II, III курсов.

Далее наш анализ был направлен на изучение психофизиологических особенностей и адаптационного потенциала студентов, имеющих признаки дефицита внимания $(n=21$ или 13,6 \% обследуемой выборки).

В таблице 4 представлены статистические данные психофизиологических показателей ПЗМР и теппинг-теста. В таблице представлены результаты по группам студентов с выявленными признаками дефицита внимания и без наличия признаков дефицита внимания, поскольку при первичном анализе статистически достоверных гендерных различий мы не выявили.

Таблицуа 4

\section{Результаты психофизиологических исследований}

Table 4

The results of psychophysiological research

\begin{tabular}{|l|c|c|c|c|}
\hline \multirow{2}{*}{\multicolumn{2}{|c|}{ Сравниваемые группы }} & \multicolumn{2}{c|}{$\begin{array}{c}\text { Теппинг-тест (число } \\
\text { ударов, кол-во) }\end{array}$} & \multicolumn{2}{c|}{ ПЗМР, мс } \\
\cline { 3 - 6 } & $\mathrm{M} \pm \delta$ & $\mathrm{CV}, \%$ & $\mathrm{M} \pm \delta$ & $\mathrm{CV}, \%$ \\
\hline $\begin{array}{l}\text { Студенты с признаками дефицита } \\
\text { внимания, } n=21\end{array}$ & $198,9 \pm 45,5$ & 22,9 & $210,5 \pm 38,8$ & 18,4 \\
\hline $\begin{array}{l}\text { Студенты без признаков дефицита } \\
\text { внимания, } n=133\end{array}$ & $193,8 \pm 23,4$ & 12,1 & $212,2 \pm 19,0$ & 8,9 \\
\hline
\end{tabular}

Представленные данные свидетельствуют об отсутствии статистически достоверных различий между показателями в разных группах обследуемых студентов. Однако показатель коэффициента вариации свидетельствует о том, что в группе студентов без признаков дефицита внимания результаты теппинг-теста имеют среднюю степень рассеивания значений, результаты ПЗМР - незначительную степень рассеивания. В группе студентов с признаками дефицита внимания показатели теппинг-теста имеют значительную степень рассеивания, показатели ПЗМР нахо- дятся в диапазоне средних значений, но приближены к значимой степени рассеивания значений.

Сумма точек теппинг-теста является характеристикой работоспособности двигательной зоны коры больших полушарий и отражает индивидуально-типологические особенности нервной системы. Согласно А. А.Ухтомскому, частота движений отражает результат усвоения ритма функциональной системой и лабильность нервно-мышечной системы.

Большой разброс показателей возбуждения и лабильности нервно-мышечной системы по показателям ПЗМР и теппинг-теста у сту- 
дентов, имеющих признаки дефицита внимания, позволяет нам предположить, что снижение свойств внимания у студентов в условиях сочетанных умственных и физических нагрузок обусловлено разными причинами. Сформированная многолетней спортивной подготовкой функциональная система имеет доминанту моторной зоны. Безусловно, для спортсмена это положительный результат. Однако в условиях перехода от физической нагрузки к умственной важно иметь хорошие качества подвижности нервных центров. К сожалению, низкая подвижность нервных центров является причиной дефицита внимания.

На диаграмме 1 представлено распределение студентов по уровням адаптационного потенциала.

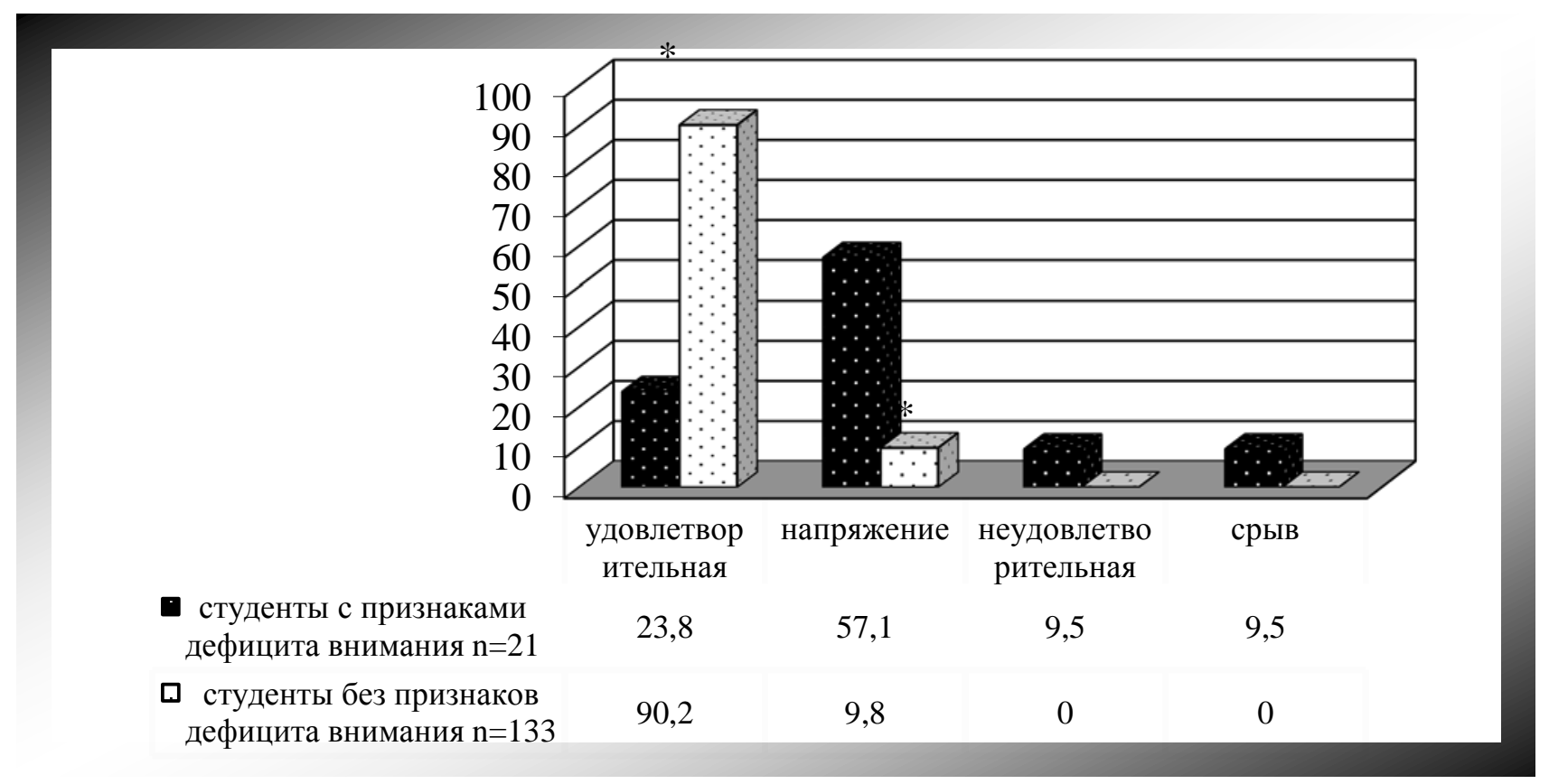

Puc. 1. Распределение студентов по уровням адаптационного потенциала, проценты Примечание: достоверность различий * $-p<0,05$; ** $-p<0,05$ по $t$-критерию Стьюдента

Fig. 1. Distribution of students by adaptational potential level, percent Note: reliability of differences: ${ }^{*}-\mathrm{p}<0,05 ; * *-\mathrm{p}<0,05$ by student's t-test

У студентов с признаками дефицита внимания наблюдается напряжение механизмов адаптации к условиям сочетанных воздействий, что характерно для $57,1 \%$ студентов этой группы. Студенты, не имеющие признаков дефицита внимания, в основном имеют удовлетворительный уровень адаптации $90,7 \%$, лишь у 9,8 \% отмечается напряжение адаптации. Среди студентов с выявленными признаками 19 \% имеют неудовлетворительный тип и срыв адаптации.
Выше обозначенный факт позволяет сделать заключение о высокой «цене» адаптации студентов - спортсменов с признаками дефицита внимания к предъявляемым нагрузкам. Предъявляемые требования без учета психофизиологических особенностей студентовспортсменов вызывают напряжение механизмов адаптации, а значит - снижение функционального состояния организма. 


\section{Обсуждение результатов}

Опираясь на литературные данные, рекомендации и пояснения к тесту Тулуз-Пьерона, мы пришли к заключению о неоднозначности трактовки результатов и необходимости дополнительного анализа скорости и точности. Согласно О. В. Поповой и соавторов, «у студентов - в случае, если скорость выполнения теста ниже 38 знаков/мин» [9, с. 80], можно предположить наличие данной патологии. При этом, обращаясь как к зарубежной, так и отечественной литературе, мы учитываем, что окончательный диагноз ставиться врачом. Часто зарубежные авторы рассматривают признаки дефицита внимания в рамках медикобиологического поля, связанного с нейроанатомическими, экспериментально-клиническими и фармакологическими доказательствами, а также в зависимости от генетической предрасположенности. В конечном итоге результатом работы является медикаментозное назначение. Эти эффекты отражают развитие эмоционально-мотивационного компонента личности, вследствие чего требуются исследовательские проекты по изучению критериев гармонизации медико-физиологических, психолого-педагогических, социальных личностных конструктов $[15 ; 16]$.

По данным Л. А. Ясюковой, низкие значения скорости работы связаны с особенностями развития оперативной памяти и визуального мышления (испытуемые постоянно устанавливают зрительный контакт с образцами на доске и на бланке), тогда как при высоких показателях скорости происходит мысленное преобразование зрительного поля, четкое выделение единого обобщающего признака (взгляд обычно совпадает с движением руки по рабочей строке, зрительного отвлечения не происходит), что определяет высокую степень развития оперативной памяти и визуального мышления.
Также мы установили, что точность выполнения теста патологически низкая. Точность, прежде всего, связана с концентрацией внимания. Также на показатель точности влияют особенности переключения внимания, объема внимания, оперативной памяти, личностных особенностей (чем выше исполнительность, ответственность, тревожность, тем выше точность исполнения теста), высокие показатели - это также и проявления устойчивости (сохранение темпа, точности на протяжении всего теста). Устойчивость характеристик тем выше, чем выше у респондента развита произвольность, настойчивость, целеустремленность. Высокая точность выявлена у 44,16 \% обследованных студентов.

Таким образом, тест Тулуз-Пьерона позволяет оценить произвольное внимание, требующее от личности сознательно регулируемого сосредоточения на предмете деятельности, т. е. целенаправленного поведения.

Известно, что физиологическая основа внимания - взаимодействие процессов возбуждения и торможения, возникающих в коре головного мозга. Это взаимодействие происходит на основе установленного И. П. Павловым закона индукции нервных процессов. Кроме того, известно, что вторая сигнальная система поддерживает возбуждение в коре головного мозга. Мозговое обеспечение контроля целенаправленного поведения обеспечивается управляющими системами мозга, которые представлены сложными многокомпонентными нейрофизиологическими механизмами [29]. Результаты исследований О. А. Семеновой, Р. И. Мачинской [30] свидетельствуют о специфической роли различных регуляторных систем мозга в формировании управляющих функций.

Воздействие сочетанных умственных и физических нагрузок на организм способ- 
ствует формированию функциональной системы, реализующейся значимой вариабельностью психофизиологических показателей и вызывающей напряжение, неудовлетворительный тип и срыв адаптации у 76,1 \% студентов с признаками дефицита внимания.

\section{Заключение}

В связи с установленными особенностями внимания контингента студентов в условиях умственной и физической деятельности, мы предлагаем придерживаться некоторых рекомендаций. Для обеспечения высокого качества произвольного внимания в учебной деятельности рекомендуем студентам использовать словесные напоминания о целях деятельности, чтение учебного материала сопровождать конспектированием, организовать рабочее место без отвлекающих раздражителей, обеспечить рациональное восстановление организма и профилактику перенапряжений, применять методы развития волевых качеств.
Преподавателям рекомендуем разработать методические средства формирования действенных мотивов учения, способствующих повышению интереса к предмету, образованию доминанты учебной деятельности, учитывать разные темпы работы студентов в группе.

Полученные нами результаты планируется дополнить рядом исследований: анализом режима дня, оценкой функционального состояния нервной системы, в том числе высшей нервной деятельности. Результаты этих исследований помогут разработать практические рекомендации по коррекции образа жизни, тренировочно-соревновательной деятельности с учетом психофизиологических характеристик личности, благодаря чему можно нивелировать негативные факторы, вызывающие напряжение функциональных систем организма студента. Используемый нами комплексный подход является одним из механизмов управления качеством образования.

\section{СПИСОК ЛИТЕРАТУРЫ}

1. Заваденко Н. Н. Синдром дефицита внимания и гиперактивности: новое в диагностике и лечении // Вестник Северного (Арктического) федерального университета. Серия: медикобиологические науки. - 2014. - № 1. - C. 31-39. URL: https://elibrary.ru/item.asp?id=21455739

2. Самарский Д. М., Бугаец Я. Е., Сосновская И. В., Трембач А. Б. Комплексный анализ невнимательности, импульсивности и гиперактивности у лиц с СДВГ юношеского и зрелого возраста // Фундаментальные исследования. - 2012. - № 9-1. - C. 65-68. URL: https://elibrary.ru/item.asp?id=17881261

3. Трембач А. Б., Бугаец Я. Е., Самарский Д. М., Толоконникова В. А. Нормативные значения невнимательности, импульсивности и гиперактивности у лиц юношеского и зрелого возраста // Физическая культура, спорт - наука и практика. - 2011. - № 1. - С. 69-74. URL: https://elibrary.ru/item.asp?id=16516516

4. Чутко Л. С., Сурушкина С. Ю., Яковенко Е. А., Никишена И. С., Анисимова Т. И. Синдром дефицита внимания у взрослых: клинико-психофизиологические проявления // Вестник Северного (Арктического) федерального университета. Серия: медико-биологические науки. - 2014. - № 1. - C. 65-71. URL: https://elibrary.ru/item.asp?id=21455743

5. Gray S. A., Fettes P., Woltering S., Mawjee K., Tannock R. Symptom Manifestation and Impairments in College Students With ADHD // Journal of Learning Disabilities. - 2016. - Vol. 49, Issue 6. - P. 616-630. DOI: https://doi.org/10.1177/0022219415576523 
6. Чутко Л. С., Сурушкина С. Ю., Яковенко Е. А., Кропотов Ю. Д., Сергеев А. В. Диагностика и лечение когнитивных нарушений при синдроме дефицита внимания у взрослых // Журнал неврологии и психиатрии им. С. С. Корсакова. - 2017. - Т. 117, № 5. - С. 9-13. URL: https://elibrary.ru/item.asp?id=29384448 DOI: https://doi.org/10.17116/jnevro2017117519-13

7. Нуреев И. Т., Циркин В. И., Злоказова М. В. Синдром дефицита внимания и гиперактивности у взрослых, его диагностика и влияние на успешность образовательной деятельности студентов (обзор литературы) // Вятский медицинский вестник. - 2012. - № 1. - С. 60-66. URL: https://elibrary.ru/item.asp?id=17660742

8. Попова О. В., Циркин В. И., Нуреев И. Т., Злоказова М. В., Трухина С. И. Влияние милдроната на состояние центральной нервной системы у студентов с признаками синдрома дефицита внимания с гиперактивностью // Вестник Нижегородского университета им. Н. И. Лобачевского. - 2010. - № 6. - C. 105-112. URL: https://elibrary.ru/item.asp?id=15613648

9. Попова О. В., Нуреев И. Т., Трухина С. И., Шушканова Е. Г., Трухин А. Н., Циркин В. И., Злоказова М. В. Особенности высших психических функций, электрической активности мозга и успешность обучения подростков и студентов с признаками СДВГ // Вятский медицинский вестник. - 2009. - № 2-4. - C. 77-81. URL: https://elibrary.ru/item.asp?id=17889612

10. Жинжило Е. В., Слепкова Н. В., Худина Ю. С., Дмитриев М. Н. Некоторые критерии диагностики СДВГ и коморбидных проявлений у студентов // Архивъ внутренней медицины. - 2016. - № S1. - C. 106-107. URL: https://elibrary.ru/item.asp?id=25910231

11. Звягина Е. В., Харина И. Ф. Интегрированный аспект изучения синдрома дефицита внимания и гиперактивности: анатомо-морфологический и психолого-педагогический ракурс // Научно-педагогическое обозрение. - 2016. - № 2 (12). - C. 46-51. URL: https://elibrary.ru/item.asp?id=26146250

12. Звягина Е. В., Харина И. Ф. Психофизиологические методы изучения свойств внимания как основа выявления синдрома дефицита внимания у студентов вуза физической культуры // Научно-педагогическое обозрение. - 2017. - № 2 (16). - C. 31-36. DOI: https://doi.org/10.23951/2307-6127-2017-2-31-36

13. Звягина Е. В., Харина И. Ф. Синдром дефицита внимания как явление у студенческой молодежи, занимающейся различными видами спортивной деятельности // Научно-спортивный вестник Урала и Сибири. - 2017. - Т. 15, № 3. - С. 52-58. URL: https://elibrary.ru/item.asp?id=29411946

14. Darcq E., Kieffer B. L. PI3K signaling in the locus coeruleus: a new molecular pathway for ADHD research // EMBO Molecular Medicine. - 2015. - Vol. 7, № 7. - P. 859-861. DOI: https://doi.org/10.15252/emmm.201505266

15. Banerjee E., Nondagopal K. Does serotonin deficit mediate susceptibility to AHD? // Neurochemistry International. - 2015. - Vol. 82. - P. 52-68. DOI: https://doi.org/10.1016/j.neuint.2015.02.001

16. Baroni A., Castellanos F. X. Stimulants, cognition and ADHD // Current Opinion in Behavioral Sciences. - 2015. - Vol. 4. - P. 109-114. DOI: https://doi.org/10.1016/j.cobeha.2015.04.010

17. Arnold L. E., Hodgkins P., Caci H., Kahle J., Young S. Effect of treatment modality on longterm outcomes in attention-deficit/hyperactivity disorder: a systematic review // PLoS One. 2015. - Vol. 10 (2). - P. e0116407. DOI: https://doi.org/10.1371/journal.pone.0116407

18. Glozman J. M., Shevchenko I. A. Executive function in children with ADHD // Psychology and Neuroscience. - 2014. - Vol. 7, № 4. - P. 453-460. DOI: https://doi.org/10.3922/j.psns.2014.4.04

19. Solovieva Y., Rojas L. Q. Syndromic analysis of ADHD at preschool according to A.R. Luria concept // Psychology and Neuroscience. - 2014. - Vol. 7, № 4. - P. 443-452. DOI: https://doi.org/10.3922/j.psns.2014.4.03 
20. Arns M., Heinrich H., Strehl U. Evaluation of neurofeedback in ADHD: the long and winding road // Biological Psychology. - 2014. - Vol. 95. - P. 108-115. DOI: https://doi.org/10.1016/j.biopsycho.2013.11.013

21. Johnson M. R. Fear of stimulant therapy for children and adolescents with attention-deficit/hyperactivity disorder // Journal of Child and Adolescent Psychopharmacology. - 2015. - Vol. 25, Issue 2. - P. 182. DOI: https://doi.org/10.1089/cap.2014.0117

22. Ozdemir S. The effects of the first step to success program on academic engagement behaviors of Turkish students with attention-deficit/hyperactivity disorder // Journal of Positive Behavior Interventions. - 2011. - Vol. 13, № 3. - P. 168-177. DOI: https://doi.org/10.1177/1098300710373503

23. Matza L. S., Devine M. K., Haynes V. S., Davies E. W., Kostelec J. M., Televantou F., J ordan J. B. Health state utilities associated with adult attention-deficit/hyperactivity disorder // Patient Preference and Adherence. - 2014. - Vol. 8. - C. 997-1006. DOI: https://doi.org/10.2147/PPA.S62776

24. Федотчев А. И., Земляная А. А., Полевая С. А., Савчук Л. В. Синдром дефицита внимания с гиперактивностью и современные возможности его лечения методом нейробиоуправления // Журнал неврологии и психиатрии им. С. С. Корсакова. - 2016. - Vol. 116, № 5. - C. 98-101. URL: https://elibrary.ru/item.asp?id=26165419 DOI: https://doi.org/10.17116/jnevro20161165198-101

25. Чутко Л. С., Сурушкина С. Ю., Анисимова Т. И. Диадный подход к лечению синдрома дефицита внимания с гиперактивностью // Журнал неврологии и психиатрии им. С. С. Корсакова. - 2017. - T. 117, № 2. - C. 81-84. URL: https://elibrary.ru/item.asp?id=28843319 DOI: https://doi.org/10.17116/jnevro20171172181-84

26. Быков Е. В., Макунина О. А., Коломиец О. И., Коваленко А. Н. Организация мониторинга состояния здоровья студентов в Уральском государственном университете физической культуры // Научно-спортивный вестник Урала и Сибири. - 2017. - Т. 13, № 1. - С. 3 9. URL: https://elibrary.ru/item.asp?id=28201220

27. Коваленко А. Н., Быков Е. В. Цели и задачи программы «Мониторинг состояния здоровья студентов университета физической культуры» // Вестник Челябинского государственного педагогического университета. - 2016. - № 9. - C. 66-71. URL: https://elibrary.ru/item.asp?id=27515512

28. Айзман Р. И. Здоровье участников образовательного процесса как критерий эффективности здоровьесберегающей деятельности в системе образования // Отечественная и зарубежная педагогика. - 2015. - № 5 (26). - C. 72-82. URL: https://elibrary.ru/item.asp?id=24346188

29. Мачинская Р. И. Управляющие системы мозга // Журнал высшей нервной деятельности им. И. П. Павлова. - 2015. - Т. 65, № 1. - C. 33. DOI: https://doi.org/10.7868/S0044467715010086 URL: https://elibrary.ru/item.asp?id=22995553

30. Семенова О. А., Мачинская Р. И. Влияние функционального состояния регуляторных систем мозга на эффективность произвольной организации когнитивной деятельности у детей. Сообщение II. Нейропсихологический и электроэнцефалографический анализ состояния регуляторных функций мозга у детей предподросткового возраста с трудностями учебной адаптации // Физиология человека. - 2015. - Т. 41, № 5. - C. 28. DOI: https://doi.org/10.1134/S0362119715050126 
DOI: $10.15293 / 2226-3365.1803 .13$

Irina Fedorovna Kharina,

Candidate of Pedagogical Sciences, Associate Professor,

Anatomy Department,

Ural State University of Physical Culture, Chelyabinsk, Russian Federation;

Research Staff Member,

Olympic Sport Scientific-Research Institute, Chelyabinsk, Russian Federation.

ORCID ID: http://orcid.org/0000-0003-0354-1841

E-mail: kharina.i.f@list.ru

Ekaterina Vladimirovna Zvyagina,

Candidate of Pedagogical Sciences, Associate Professor,

Anatomy Department,

Ural State University of Physical Culture, Chelyabinsk, Russian Federation;

Research Staff Member,

Olympic Sport Scientific-Research Institute, Chelyabinsk, Russian Federation.

ORCID ID: http://orcid.org/0000-0001-8808-1148

E-mail: zv-aev@mail.ru

Evgeny Vital'evich Bykov,

Doctor of Medical Sciences, Professor, Head, Vice-Rector for Research,

Sport Medicine Department,

Ural State University of Physical Culture, Chelyabinsk, Russian Federation.

ORCID ID: http://orcid.org/0000-0002-7506-8793

E-mail: bev58@yandex.ru

Olga Alexandrovna Makunina,

Candidate of Biological Sciences, Associate Professor,

Physiology Department,

Ural State University of Physical Culture, Chelyabinsk, Russian Federation;

Research Staff Member,

Olympic Sport Scientific-Research Institute, Chelyabinsk, Russian Federation.

ORCID ID: http://orcid.org/0000-0002-3448-9428

E-mail: oamakunina@mail.ru

\title{
Psychophysiological characteristics of students with the symptoms of attention deficit disorder under the conditions of combined mental and physical loads
}

\begin{abstract}
Introduction. Students, having signs of attention deficit differ by psychophysiological features, especially necessary in conditions of combined mental and physical activities. This article presents the research results of the psychophysiological characteristics in University of Physical Culture students with signs of attention deficit. The aim of the research is to study psychophysiological peculiarities and adaptation of University of Physical Culture students with signs of attention deficit disorder.

Materials and Methods. The research was conducted using the Toulouse-Pieron test, which is one of the variants of the correction task, the general principle of which is to differentiate the stimuli, similar in form and content for a precisely defined time. Firstly, attention characteristics (concentration,
\end{abstract}


stability, shifting) measured are measured, secondly, the accuracy and reliability of information processing. 154 students (I-III years) were examined. Physiological parameters were evaluated by the simple hand-eye reaction and tapping-test. Adaptation assessment was assessed by means of R. M. Bayevsky's formula.

Results. It was found, that students' working speed did not correspond to age norms: the research revealed low speed in 54 students (35,06\%); 70 students (45,45\%) showed average speed. The authors identified signs of attention deficit disorder in $1^{\text {st }}$ and $2^{\text {nd }}$ year students (8,82\% and 9,62 \% respectively). 44,16 \% (68 students) showed high accuracy level. The students' psychophysiological characteristics correspond to age norms and do not have any differences, depending on attention characteristics, between students. But significant variance of psychophysiological characteristics variation coefficients can indicate different causes of students' attention deficit. Students, having signs of attention deficit experience adaptation stress.

Conclusions. Some recommendations for students and teachers aimed at increasing learning outcomes are presented in the article. The research will be continued in the context of discovering reasons for attention deficit disorder and studying students' neurophysiological peculiarities under the conditions of combined mental and physical loads.

\section{Keywords}

Health monitoring; Attention deficiency syndrome; Attention peculiarities; Students-sportsmen; Toulouse-Pierron test; Adaptation; Psychophysiological features.

\section{Acknowledgements}

The research was supported by Ministry of Sports of Russian Federation, State Task "The Ural State University of Physical Culture (Chelyabinsk, Russian Federation) students' health monitoring”

\section{REFERENCES}

1. Zavadenko N. N. Attention Deficit Hyperactivity Disorder: New Developments in Diagnosis and Treatment. Bulletin of Northern (Arctic) Federal University. Series Medical and Biological Sciences, 2014, no. 1, pp. 31-39. (In Russian) URL: https://elibrary.ru/item.asp?id=21455739

2. Samarskiy D. M., Bugaez J. E., Sosnovskaya I. V., Trembach A. B. The Complex Analysis of the Inattention, Impulsivity and Hyperactivity in Adolescent and Young Adults with ADHD. Fundamental Research, 2012, no. 9-1, pp. 65-68. (In Russian) URL: https://elibrary.ru/item.asp?id=17881261

3. Trembach A., Bugaets Yu., Samarskii D., Tolokonnikov V. Normative Meaning of Inattentiveness, Impulsiveness and Hyperactivity in Youth and Mature Aged Persons. Physical Education, Sports - Science and Practice, 2011, no. 1, pp. 69-74. (In Russian) URL: https://elibrary.ru/item.asp?id=16516516

4. Chutko L. S., Surushkina S. Yu., Yakovenko E. A., Nikishena I. S., Anisimova T. I. Attention Deficit Disorder in Adults: Clinical And Psychophysiological Manifestations. Bulletin of Northern (Arctic) Federal University. Series Medical and Biological Sciences, 2014, no. 1, pp. 65-71. (In Russian) URL : https://elibrary.ru/item.asp?id=21455743

5. Gray S. A., Fettes P., Woltering S., Mawjee K., Tannock R. Symptom Manifestation and Impairments in College Students With ADHD. Journal of Learning Disabilities, 2016, vol. 49, issue 6, pp. 616-630. DOI: https://doi.org/10.1177/0022219415576523

6. Chutko L. S., Surushkina S. Yu., Yakovenko E. A., Kropotov Yu. D., Sergeev A. V. Diagnosis and treatment of cognitive impairment in adults with attention deficit hyperactivity disorder. 
S. S. Korsakov Journal of Neurology and Psychiatry, 2017, vol. 117, no. 5, pp. 9-13. (In Russian) https://elibrary.ru/item.asp?id=29384448 DOI: https://doi.org/10.17116/jnevro2017117519-13

7. Nureev I. T., Tzirkin V. I., Zlokazova M. V. Adult attention deficit hyperactivity disorder, diagnosis and its and impact on the success of the educational activities of students (review). Medical Newsletter of Vyatka, 2012, no. 1, pp. 60-66. (In Russian) URL: https://elibrary.ru/item.asp?id=17660742

8. Popova O. V., Tsirkin V. I., Nureev I. T., Zlokazova M. V., Trukhina S. I. The Influence of Mildronate on the Condition of Central Nervous System of Students with Attention Deficit Hyperactivity Disorder (ADHD) Syndrome. Bulletin of Lobachevsky University of Nizhni Novgorod, 2010, no. 6, pp. 105-112. (In Russian) URL: https://elibrary.ru/item.asp?id=15613648

9. Popova O. V., Nureev I. T., Trukhina S. I., Shushkanova E. G., Trukhin A. N., Tzirkin V. I., Zlokazova M. V. Features of the supreme mental functions, brain electric activity and success of training of teenagers and students with ADHD. Medical Newsletter of Vyatka, 2009, no. 2-4, pp. 77-81. (In Russian) URL: https://elibrary.ru/item.asp?id=17889612

10. Zhinzhilo E. V., Slepkova N. V., Khudina J. S., Dmitriev M. N. Some Criteria of Diagnostics of Syndrome of ADHD and Comorbide Implications at Students. Russian Archives of Internal Medicine, 2016, no. S1, pp. 106-107. (In Russian) URL: https://elibrary.ru/item.asp?id=25910231

11. Zvyagina E. V., Kharina I. F. Integrated Aspect of Attention Deficit Disorder and Hyperactivity Study: Anatomic-Morphological and Psychological Angles. Pedagogical Review, 2016, no. 2, pp. 46-51. (In Russian) URL: https://elibrary.ru/item.asp?id=26146250

12. Zvyagina E. V., Kharina I. F. Psychophysiological Methods of Studying of Properties of Attention as the Basis for Identification of Attention Deficit Syndrome of Students of Higher Education Institution of Physical Culture. Pedagogical Review, 2017, no. 2, pp. 31-36 (In Russian) URL: https://elibrary.ru/item.asp?id=29109118 DOI: https://doi.org/10.23951/2307-6127-2017-2-31-36

13. Zvyagina E. V., Kharina I. F. The Attention Deficit Disorder as a Phenomenon in Students Engaged in Various Kinds of Sports Activities. Ural and Siberia Bulletin of Sports Science, 2017, vol. 15, no. 3, pp. 52-58. (In Russian) URL: https://elibrary.ru/item.asp?id=29411946

14. Darcq E., Kieffer B. L. PI3K signaling in the locus coeruleus: a new molecular pathway for ADHD research. EMBO Molecular Medicine, 2015, vol. 7, no. 7, pp. 859-861. DOI: https://doi.org/10.15252/emmm.201505266

15. Banerjee E., Nondagopal K. Does serotonin deficit mediate susceptibility to AHD? Neurochemistry International, 2015, vol. 82, pp. 52-68. DOI: https://doi.org/10.1016/j.neuint.2015.02.001

16. Baroni A., Castellanos F. X. Stimulants, cognition and ADHD. Current Opinion in Behavioral Sciences, 2015, vol. 4, pp. 109-114. DOI: https://doi.org/10.1016/j.cobeha.2015.04.010

17. Arnold L. E., Hodgkins P., Caci H., Kahle J., Young S. Effect of treatment modality on long-term outcomes in attention-deficit/hyperactivity disorder: a systematic review. PLoS One, 2015, vol. 10 (2), pp. e0116407. DOI: https://doi.org/10.1371/journal.pone.0116407

18. Glozman J. M., Shevchenko I. A. Executive function in children with ADHD. Psychology and Neuroscience, 2014, vol. 7, no. 4, pp. 453-460. DOI: https://doi.org/10.3922/j.psns.2014.4.04

19. Solovieva Y., Rojas L. Q. Syndromic analysis of ADHD at preschool according to A.R. Luria concept. Psychology and Neuroscience, 2014, vol. 7, no. 4, pp. 443-452. DOI: https://doi.org/10.3922/j.psns.2014.4.03

20. Arns M., Heinrich H., Strehl U. Evaluation of neurofeedback in ADHD: the long and winding road. Biological Psychology, 2014, vol. 95, pp. 108-115. DOI: https://doi.org/10.1016/j.biopsycho.2013.11.013 
21. Johnson M. R. Fear of stimulant therapy for children and adolescents with attentiondeficit/hyperactivity disorder. Journal of Child and Adolescent Psychopharmacology, 2015, vol. 25, issue 2, pp. 182. DOI: https://doi.org/10.1089/cap.2014.0117

22. Ozdemir S. The effects of the first step to success program on academic engagement behaviors of Turkish students with attention-deficit/hyperactivity disorder. Journal of Positive Behavior Interventions, 2011, vol. 13, no. 3, pp. 168-177. DOI: https://doi.org/10.1177/1098300710373503

23. Matza L. S., Devine M. K., Haynes V. S., Davies E. W., Kostelec J. M., Televantou F., Jordan J. B. Health state utilities associated with adult attention-deficit/hyperactivity disorder. Patient Preference and Adherence, 2014, vol. 8, pp. 997-1006. DOI: https://doi.org/10.2147/PPA.S62776

24. Fedotchev A. I., Zemlyanaya A. A., Polevaya S. A., Savchuk L. V. Attention deficit hyperactivity disorder and current possibilities of its treatment by the method of neurofeedback training. S. S. Korsakov Journal of Neurology and Psychiatry, 2016, vol. 116, no. 5, pp. 98101. (In Russian) https://elibrary.ru/item.asp?id=26165419 DOI: https://doi.org/10.17116/jnevro20161165198-101

25. Chutko L. S., Surushkina S. Yu., Anisimova T. I. A dyad approach to the treatment of attention deficit hyperactivity disorder. S. S. Korsakov Journal of Neurology and Psychiatry, 2017, vol. 117, no. 2, pp. 81-84. (In Russian) https://elibrary.ru/item.asp?id=28843319 DOI: https://doi.org/10.17116/jnevro20171172181-84

26. Bykov E. V., Makunina O. A., Kolomiets O. I., Kovalenko A. N. Organization of Monitoring Health Students at the Ural State University of Physical Culture. Ural and Siberia Bulletin of Sports Science, 2017, vol. 13, no. 1, pp. 3-9. (In Russian) URL: https://elibrary.ru/item.asp?id=28201220

27. Kovalenko A. N., Bykov E. V. A goals and objectives of the program "Health monitoring of students at the university of physical education". Herald of Chelyabinsk State Pedagogical University, 2016, no. 9, pp. 66-71. (In Russian) URL: https://elibrary.ru/item.asp?id=27515512

28. Aizman R. I. Health And Safety Of Participants Of Educational Process Is A Criterion Of HealthSaving Activity Efficacy At The Educational System. National and Foreign Pedagogica, 2015, no. 5, pp. 72-82. (In Russian) URL: https://elibrary.ru/item.asp?id=24346188

29. Machinskaya R. I. The Brain Executive Systems. Zh Vyssh Nerv Deiat I P Pavlova, 2015, vol. 65, no. 1, pp. 33. (In Russian) DOI: https://doi.org/10.7868/S0044467715010086 URL: https://elibrary.ru/item.asp?id=22995553

30. Semenova O. A., Machinskaya R. I. The influence of the functional state of brain regulatory systems on the efficiency of voluntary regulation of cognitive activity in children: II. Neuropsychological and EEG analysis of brain regulatory functions in 10-12-year-old children with learning difficulties. Human Physiology, 2015, vol. 41, no. 5, pp. 478-486. DOI: https://doi.org/10.1134/S0362119715050126

Submitted: 15 March 2018 Accepted: 11 May 2018 Published: 30 June 2018

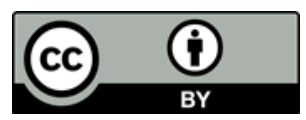

This is an open access article distributed under the Creative Commons Attribution License which permits unrestricted use, distribution, and reproduction in any medium, provided the original work is properly cited. (CC BY 4.0). 